\title{
Reaction to spatial novelty and exploratory strategies in baboons
}

\author{
S. GOUTEUX, J. VAUCLAIR, and C. THINUS-BLANC \\ Centre National de la Recherche Scientifique, Marseille, France
}

\begin{abstract}
Exploratory activity was examined in 4 young baboons with the aim of investigating the type of spatial coding (purely geometric and/or by taking into account the identity of the object) used for the configuration of objects. Animals were individually tested in an outdoor enclosure for their exploratory reactions (contact time and order of spontaneous visits) to changes brought about to a configuration of different objects. Two kinds of spatial changes were made: a modification (1) of the shape of the configuration (by displacement of one object) and (2) of the spatial arrangement without changing the initial shape (exchanging the location of two objects). In the second experiment, the effect of a spatial modification of the global geometry constituted by four identical objects was investigated. Finally, in the third experiment, a substitution of a familiar object with a novel one was performed without changing the objects' configuration. The baboons strongly reacted to geometrical modifications of the configuration. In contrast, they were less sensitive to modifications of local features that did not affect the initial spatial configuration. Analyses of spontaneous exploratory activities revealed two types of exploratory strategies (cyclic and back-and-forth). These data are discussed in relation to (1) the distinction between the encoding of geometric versus local spatial features and (2) the spatial function of exploratory activity.
\end{abstract}

Reaction to novelty is a behavior frequently observed in many mammalian species when they are confronted with a change in their physical environment. For example, a new object added to a series of familiar objects is selectively explored in preference to the familiar objects. Such effects, called reactions to change, have been observed in rats (Berlyne, 1960), gerbils (Cheal, 1978), marmosets (Menzel \& Menzel, 1979), and baboons (Joubert \& Vauclair, 1986). Furthermore, mammals are also able to detect a specific change of location of one or more familiar objects that have been set up in an open field. These reactions to spatial novelty have been found in rats (Corman \& Shafer, 1968), gerbils (Cheal, 1978), and hamsters (Poucet, Chapuis, Durup, \& Thinus-Blanc, 1986; Thinus-Blanc et al., 1987). Animals reacted to the spatial modification by a renewal of investigatory responses specifically directed toward the displaced or the new object(s). If the modification does not induce an increase of the exploratory activity, it may be concluded that the feature of the situation that has been affected by the modification has not been encoded in the representa-

This research was supported by the Centre National de la Recherche Scientifique and by the Ministère de l'Education Nationale, de l'Enseignement Supérieure et de la Recherche (Grant 97-5-03224). The authors thank P. Lucciani and all the staff of the Primatology Unit in Rousset (France). In addition, we thank E. Damerose, G. Ferreira, M. Steadt, and P. A. Stis for comments, suggestions, and assistance with the experiments and for useful comments on an earlier version of this manuscript. Correspondence should be addressed to S. Gouteux, Center for Research in Cognitive Neurosciences, National Center for Scientific Research (CNRS), 31 Chemin Joseph Aiguier, 13402 Marseille Cedex 20, France (e-mail: gouteux@Inf.cnrs-mrs.fr). tion of the animal, or that the change has not been noticed by the animal.

In the realm of spatial cognition, comparisons of renewed exploratory reactions directed toward displaced and nondisplaced objects provide further insight about the spatial information that is (or is not) encoded in the internal representation of the animal. Reaction to spatial change is taken as reflecting the fact that the animal has built some internal representation or a "cognitive map" (Tolman, 1948) of the familiar spatial situation that is later compared with the unfamiliar situation during the test. Thus, the study of spatial exploration of a configuration of objects may help to better understand the elements that constitute spatial representations, and especially the type of relationships that the objects establish among these elements, and that are stored in memory.

Other approaches have also been used to investigate spatial representations and cognitive mapping in animals. For example, Tinklepaugh (1932) used a delayed-reaction task with monkeys. The task required the animals to discriminate between two cups located in different locations, one of which had previously been baited with food. Tinklepaugh found that monkeys were very accurate in this task, but that the accuracy declined when the task was made more complex by increasing the number of cup pairs. Similarly, Menzel (1973) found that chimpanzees that had seen food hidden in 18 locations were more accurate in retrieving that food than were control animals that had not seen the baited sites. Moreover, according to Menzel, the chimpanzees tended to use a "least-distance" strategy to minimize the total distance traveled to retrieve the hidden food. More recently, Cramer and Gallistel 
(1997) came to the same conclusion in a study with vervet monkeys. In their study, the monkeys were carried around a square enclosure to watch where food rewards were hidden. Like Menzel's chimpanzees, the vervet monkeys tended to minimize the distance traveled by ignoring the order of baiting. Furthermore, Cramer and Gallistel found that the choice of the next destination (a baited site) was determined by at least two subsequent destinations.

Other nonhuman primates have been tested in similar tasks. For example, MacDonald (1994), MacDonald and Wilkie (1990), and MacDonald, Pang, and Gibeault (1994) demonstrated that during foraging behavior in a delayed food recovery task in which several sites had been baited, gorillas, yellow-nosed monkeys, and marmosets minimized the total distance traveled between sites. They may have used a least-distance strategy to perform the task. The same interpretation has been proposed by De Lillo, Visalberghi, and Aversano (1997) in a study with capuchin monkeys. The authors showed that the food-searching strategies of capuchin monkeys in an apparatus featuring a set of baited containers were structured in a such a way as to reduce not only running distance but also the memory demands of the task.

Thus, from previous works in nonhuman primates, it can be concluded that apes and monkeys possess a spatial memory and cognitive capacities that allow them to optimize their food-searching behavior. To our knowledge, no study has tested the reactions of nonhuman primates to changes in the spatial arrangement of a set of familiar objects. Even when other methods are used, little is known about the spatial characteristics encoded in the spatial representations of nonhuman primates compared with what is known from studies conducted with rodents or birds in the same domain (see, e.g., Brodbeck, 1994). Furthermore, unlike human studies, no investigation has been conducted so far with the specific aim of detecting and analyzing locomotor regularities during spatial exploration in nonhuman primates. For example, in humans, Gaunet and Thinus-Blanc (1996) have demonstrated the existence of two main patterns of exploration during a free exploration of four objects located in a room (the locomotor space): "cyclic patterns," which involve consecutive visits to all of the objects, and "back-andforth" patterns, which include several consecutive visits to the same objects of a pair. According to the authors and to Neisser's (1976) theory, those exploration strategies might reflect some preexisting schemata that control the organization of information gathering. Thus, the study of patterns of exploration in nonhuman primates may help to better understand the mechanisms involved in the construction and use of spatial representations.

By using the two aforementioned methods (reaction to spatial change and investigation of locomotor regularities), our first objective was to analyze the spatial characteristics of an object's configuration encoded by baboons. Our second objective was to study the exploration patterns (if any) displayed by the baboons in order to detect possible locomotor strategies during exploration. To meet both objectives, we analyzed in three experiments the exploratory behavior of 4 nonhuman primates (baboons) confronted with spatial modifications of an object's configuration. In the first experiment, we investigated the effects of changes in the geometry of the configuration composed of four different objects. The objects were not directly perceptible, but hidden in identical boxes. Two different spatial changes were made: a modification of the shape of the configuration (displacement of one object) and a modification of the spatial arrangement without changing the initial shape (permutation of two objects). In the second experiment, a spatial modification of the global geometry, constituted by four identical objects, was investigated. Finally, in the third experiment, we observed the baboon's behavior when these animals were confronted with a nongeometrical modification of the configuration of objects (substitution of a familiar object by a novel one).

\section{EXPERIMENT 1}

\section{Method}

Animals. Four experimentally naive sub-adult baboons (Papio anubis) living in a social group were individually studied. The group comprised 3 males (Idefix, Ignace, and Jason) and 1 female (Jade). All animals were about 3.5 years of age at the beginning of the experiment. All baboons were born in captivity at the Primatology Unit of the CNRS in Rousset (France). They were housed together in the enclosure described below 1 year before the beginning of the experiment. They received food pellets and vegetables in their enclosure once a day in the morning.

Apparatus. The enclosure consisted of two parts: an indoor enclosure and an outdoor enclosure. The outdoor part was used as the test environment and had a total area of $24.75 \mathrm{~m}^{2}$. A tunnel connected the outdoor enclosure to the indoor enclosure (a concrete building of $6.75 \mathrm{~m}^{2}$ ). During the test period, animals were not permitted to move freely from one area to the other. The test environment contained a configuration of four objects (Figure 1): Object A was a tennis ball, Object B was a brown rectangular metal tube $(10 \times 5 \mathrm{~cm})$, Object $\mathrm{C}$ was a blue Plexiglas disk $(10 \mathrm{~cm}$ in diameter $)$, and Object $D$ was a red plastic pipe $(10 \times 5 \mathrm{~cm})$. These objects were placed in four opaque identical boxes $(15 \times 25 \mathrm{~cm})$ so that the animal could not identify the objects at distance. Once respective position of each object and its box was chosen, they were left in the same position for the entire duration of Experiment 1 (except during the test phase).

The boxes were attached to three of the four walls of the test environment at least $1 \mathrm{~m}$ from each other so as to prevent animals from exploring them in a systematic way.

Data Collection and Analysis. The data collected from each session were the number and duration of contacts with each object. A contact was defined as the animal's hand or mouth actually touching an object (a contact with only the containing box did not count). A silent stopwatch was used to measure contact duration. A camera was placed $10 \mathrm{~m}$ from the experimental environment, and each session was recorded to later verify contact duration measurements made during the experiments and to analyze exploratory patterns.

The following variables were recorded: (1) the total number of objects visited, which provides an index of the global level of exploratory activity, and (2) the number of visits to the four different 


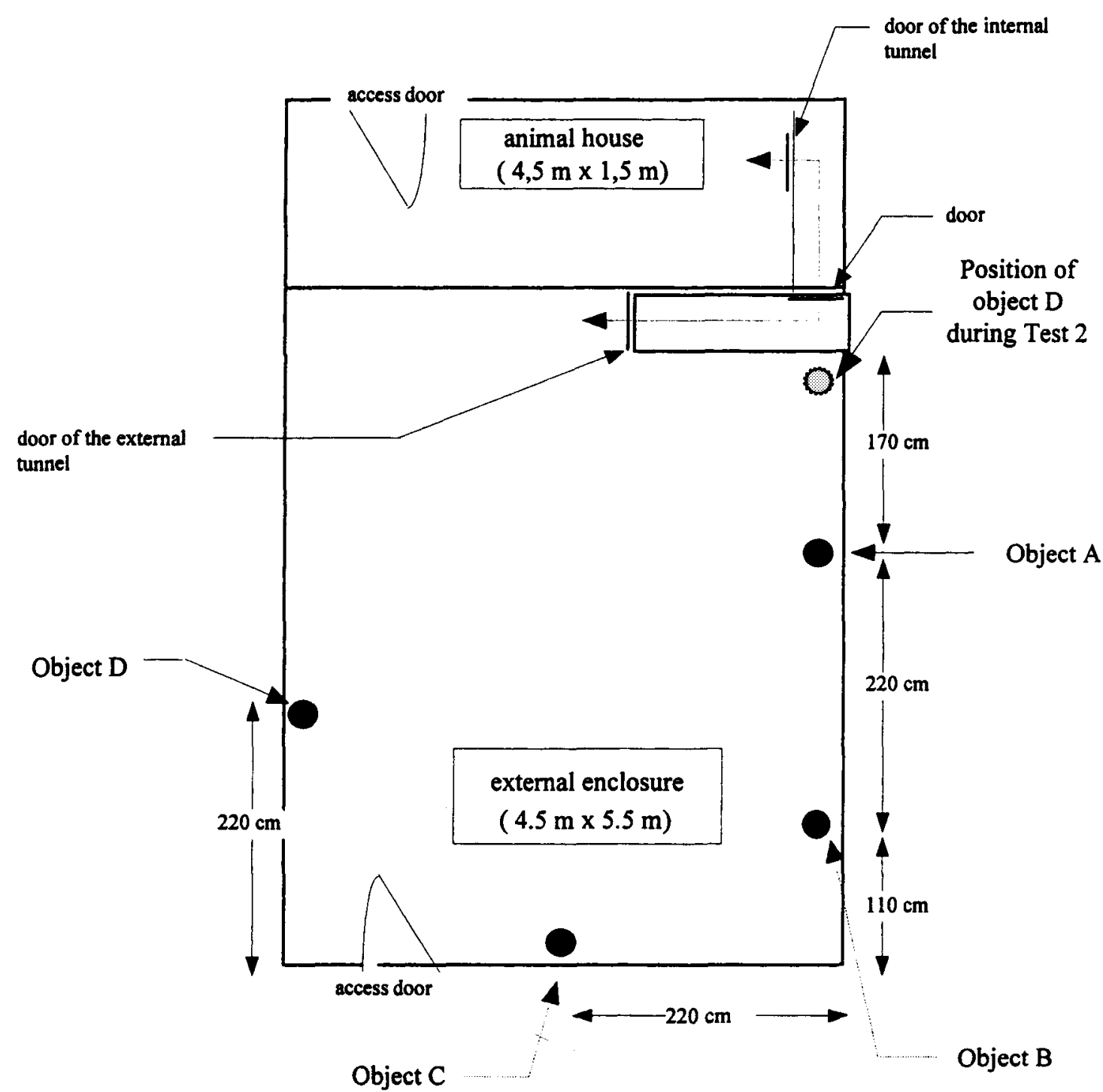

Figure 1. Illustration of the experimental environment during the initial configuration of Experiment 1 showing the positions of the four identical boxes (black circles), each containing one object. The gray circle represents the new location of Object D during Test 2 (Experiment 1).

objects, in order to ascertain that none of them raised a particular interest that might have biased the reaction-to-change detection. Given that the analyses conducted on the number and duration of contacts yielded comparable results, only the results concerning duration will be presented here.

Because one of the aims of this experiment was to investigate whether systematic patterns of exploration would be displayed by the animals, the sequences of visits to the places during each exploratory session were analyzed with a computer program. For that purpose, one letter was assigned to each object so that sequences of letters corresponded to sequences of visits to objects. The program was designed to isolate and record several types of patterns that have been found previously in humans (Gaunet \& Thinus-Blanc, 1996). The maximum tolerated overlap between the two categories of exploratory patterns corresponds to one place. For example, in the sequence of visits to the places ACBDADAD, the second visit to $A$ (underlined) was considered as belonging both to a cyclic
(ACBDA) and to a back-and-forth (ADAD) pattern. For each animal, the total number of objects included in the whole sequence and in the sequences corresponding to the above-defined exploratory patterns was computed. For instance, if three back-and-forth patterns including four visits, and two cyclic patterns including five visits were recorded, then the total number of visited places was $(3 \times 4)$ $+(2 \times 5)=24$ with $3 \times 4=12$ for back-and-forth and $2 \times 5=10$ for cyclic patterns.

Procedure. The basic procedure was the same for all animals and for all the experiments. Baboons were tested individually. A preexperimental phase of 2 months was first conducted to familiarize the animals with isolation and with the presence of new objects in their familiar environment. Each animal was thus isolated daily in the outside enclosure for $1 \mathrm{~h}$, and then fed. Progressively, after feeding, some boxes were randomly attached to the walls and some objects were added in the external zone. Baboons had the opportunity to manipulate these objects for $10 \mathrm{~min}$. The objects, but not the 
boxes, were all different from those used in the experiments. During the preexperimental phase, animals also learned to exit from the indoor enclosure and to run into the center of the outdoor enclosure where a reward (food pellets) was to be found. Thus, the start position of the animals was controlled.

Each experiment was divided into three phases: a habituation phase, a test phase, and a control phase. During those three phases, sessions were conducted each day from 10 a.m. to 3 p.m. The habituation phase (Days 1 and 2) allowed the animal to become familiar with the initial spatial configuration made by the four objects. In this phase, the animal was introduced into the experimental enclosure and could freely explore the configuration for $10 \mathrm{~min}$ (Session 1). Then the baboon was taken back into the indoor enclosure for $1 \mathrm{~h}$ before starting another 10 -min exploration of the configuration (Session 2).

The test phase (Days 3 and 4) was aimed at examining the animal's reaction toward a new spatial configuration of the same objects. Two manipulations were performed: On Day 3 (Test 1), a permutation of Objects $A$ and $D$ was performed without changing the shape of the initial configuration. On Day 4 (Test 2), Object D only was displaced (to the new location, as indicated in Figure 1) to induce a modification of the shape of the initial configuration. The exploration time was the same for Tests 1 and 2, as during the habituation phase $(10 \mathrm{~min})$. The order in which the animals were tested was randomly chosen for Tests 1 and 2 . Before each test session, one 10-min control session was performed with the initial configuration. This control of the habituation level was conducted $1 \mathrm{~h}$ before the beginning of the test.

A control phase was conducted in order to determine whether the different tests had interfered with the representation of the initial configuration. Thus, on Day 5 , baboons were confronted with the initial configuration during two sessions of $10 \mathrm{~min}$ each, separated from each other by a period of $1 \mathrm{~h}$.

\section{Results}

Preliminary comment. Given the limited number of the tested subjects $(N=4)$ and of behavioral observations, the use of statistical tests (parametrical or nonparametrical) was judged as inadequate. However, for habituation, we set an arbitrary criterion: A measure during the habituation phase was considered to be meaningful at the group level, and not randomly determined if all animals reacted in the same way. In addition, and as indication, a paired $t$ test was calculated in the first and last sessions of the habituation phase. For the analysis of the data during the test sessions, we set a second criteria: If there was a ratio of $3: 1$ between duration of contact times for nondisplaced and displaced objects, the result was considered meaningful.

Habituation. During the habituation phase, the mean duration (in seconds) of contacts per object and per session for all the animals decreased from $23.13 \mathrm{sec}(S D=$ $11.02 \mathrm{sec}$; Day 1, Session 1, hereafter denoted as D1-S1) to $2.31(S D=2.14 \mathrm{sec})$ at the end of the habituation phase [D2-S2; paired $t$ test, $t(15)=6.27, p<.001]$. Furthermore, the mean duration of contacts per object was always longer for the first session (D1-S1:23.13, SD = $11.02 \mathrm{sec}$; D2-S1: $9.69, S D=5.15 \mathrm{sec}$ ) than for the second (D1-S2: $8.81, S D=5.19 \mathrm{sec} ; \mathrm{D} 2-\mathrm{S} 2: 2.31, S D=2.14 \mathrm{sec})$.

Test 1 (exchange of Objects $A$ and $D$ ). The mean duration of contact per object was $5.81(S D=3.91 \mathrm{sec})$. This result was close to the mean duration of contact ob- tained in the habituation-control session - that is, the first session of Day 3 (D3-S1: $5.19, S D=3.21 \mathrm{sec}$ ).

Test 2 (displacement of Object D). The mean duration of contact for the nondisplaced objects was 6.67 $(S D=4.89 \mathrm{sec})$, while it was $79.00(S D=26.00 \mathrm{sec})$ for the displaced object. Thus, the mean contact time with the displaced objects represents 12.3 times the mean contact time with the nondisplaced objects.

Control. The mean durations of contacts per object for Sessions D5-S1 and D5-S2 were, respectively, 4.19 $(S D=2.73 \mathrm{sec})$ and $4.63(S D=5.27 \mathrm{sec})$. Individual results are presented in Table 1.

Table 1

Experiment 1: Individual Results (Contact Time per Object in Seconds) for the 4 Subjects (Idefix, Ignace, Jade, and Jason)

\begin{tabular}{|c|c|c|c|c|}
\hline \multirow[b]{2}{*}{ Day-Session (D-S) } & \multicolumn{4}{|c|}{ Object } \\
\hline & $\mathrm{A}$ & $\mathrm{B}$ & $\mathrm{C}$ & $\mathrm{D}$ \\
\hline \multicolumn{5}{|c|}{ Idefix } \\
\hline $1-1$ & 27 & 18 & 46 & 28 \\
\hline $1-2$ & 16 & 22 & 13 & 21 \\
\hline $2-1$ & 8 & 1 & 4 & 3 \\
\hline $2-2$ & 2 & 4 & 1 & 0 \\
\hline $3-1$ & 10 & 7 & 1 & 6 \\
\hline $3-2^{*}$ & 7 & 2 & 2 & 12 \\
\hline $4-1$ & 9 & 4 & 3 & 8 \\
\hline $4-2^{*}$ & 2 & 4 & 2 & 130 \\
\hline $5-1$ & 5 & 1 & 1 & 7 \\
\hline $5-2$ & 11 & 17 & 0 & 3 \\
\hline \multicolumn{5}{|c|}{ Ignace } \\
\hline $1-1$ & 16 & 13 & 17 & 10 \\
\hline $1-2$ & 5 & 7 & 10 & 4 \\
\hline $2-1$ & 9 & 14 & 7 & 0 \\
\hline $2-2$ & 0 & 4 & 1 & 3 \\
\hline $3-1$ & 3 & 4 & 3 & 8 \\
\hline $3-2^{*}$ & 5 & 6 & 4 & 5 \\
\hline $4-1$ & 7 & 6 & 4 & 8 \\
\hline $4-2^{*}$ & 4 & 5 & 4 & 44 \\
\hline $5-1$ & 3 & 5 & 4 & 11 \\
\hline $5-2$ & 0 & 4 & 2 & 2 \\
\hline \multicolumn{5}{|c|}{ Jade } \\
\hline $1-1$ & 14 & 12 & 18 & 2 \\
\hline $1-2$ & 3 & 9 & 0 & 1 \\
\hline $2-1$ & 9 & 5 & 11 & 11 \\
\hline $2-2$ & 2 & 7 & 0 & 0 \\
\hline $3-1$ & 0 & 1 & 4 & 2 \\
\hline $3-2^{*}$ & 2 & 0 & 0 & 6 \\
\hline $4-1$ & 1 & 0 & 5 & 5 \\
\hline $4-2^{*}$ & 20 & 8 & 9 & 62 \\
\hline $5-1$ & 8 & 0 & 0 & 11 \\
\hline $5-2$ & 0 & 0 & 0 & 0 \\
\hline \multicolumn{5}{|c|}{ Jason } \\
\hline $1-1$ & 41 & 36 & 41 & 31 \\
\hline $1-2$ & 12 & 9 & 4 & 5 \\
\hline $2-1$ & 24 & 13 & 23 & 13 \\
\hline $2-2$ & 5 & 0 & 0 & 8 \\
\hline $3-1$ & 9 & 8 & 3 & 14 \\
\hline $3-2^{*}$ & 16 & 1 & 15 & 10 \\
\hline $4-1$ & 4 & 3 & 5 & 6 \\
\hline $4-2^{*}$ & 19 & 1 & 33 & 80 \\
\hline $5-1$ & 4 & 2 & 3 & 2 \\
\hline $5-2$ & 3 & 2 & 0 & 4 \\
\hline
\end{tabular}

*Test Sessions. 


\section{Discussion}

During the habituation phase, the decrease of the duration of contacts indicates that the baboons got progressively familiarized with the initial situation. Test 1 (objects permutation, without changing the shape of the initial configuration) did not induce a selective reexploration of the permuted objects. In contrast, during Test 2 (modification of the initial shape by the displacement of an object), a selective reexploration directed toward the displaced object only was observed. Results of the control phase indicate that testing conditions did not affect the recollection of the initial configuration by the baboons.

The massive reexploration of the displaced object during Test 2 indicates that the baboons reacted strongly to the configuration made by the four boxes. Thus, in that experiment, novelty detection appears to have been bounded to the shape of the configuration made by the four boxes - that is, by the geometrical aspect of the configuration. The local aspect (the position of the object enclosed in the boxes) does not seem to be a crucial component of this spatial coding. To test this hypothesis, we suppressed, in the second experiment, the variations of these local characteristics by making the contents of the different boxes uniform.

\section{EXPERIMENT 2}

The purpose of this experiment was to determine the effects of a spatial modification of an arrangement made by four identical boxes, each containing an identical object. In this experimental setup, the modification uniquely affected the geometry of the global arrangement.

\section{Method}

Animals. The animals were the same 4 baboons (Papio anubis) as in Experiment 1.
Apparatus. The apparatus was identical to that used in Experiment 1 . The objects chosen were four identical orange plastic pipes $(11 \times 4 \mathrm{~cm})$.

Procedure. The basic procedure of Experiment 2 was the same as in the previous experiment except that during the test phase (Day 3), one box (Box B) was displaced to a new location that modified the geometry of the configuration. The positions of the four objects, randomly chosen, were all different from those in Experiment 1 , thus forming a new geometrical configuration (Figure 2). Experiment 2 was conducted 2 months after completion of Experiment 1 in order to reduce, as much as possible, interexperiment interference.

\section{Results}

Habituation. As in the previous experiment, the mean duration (in seconds) of contacts per object and per session for all the animals decreased from $26.81(S D=$ $4.59 \mathrm{sec})$ at D $1-\mathrm{S} 1$ to $3.81[S D=2.16 \mathrm{sec} ; t(15)=14.5$, $p<.001]$ at the end of the habituation session. Furthermore, the mean duration of contacts per object was always higher for the first session (D1-S1: $26.81, S D=4.59 \mathrm{sec}$; D2-S1: $7.88, S D=2.89 \mathrm{sec}$ ) than for the second (D1-S2: $13.44, S D=3.74 \mathrm{sec}$; D2-S2: $3.81, S D=2.38 \mathrm{sec}$ ).

Test 1 (one object displaced). The mean duration of contact per object was $4.06(S D=2.95 \mathrm{sec})$. That result was similar to the mean duration of contact observed at the end of the habituation phase (D3-S1: $6.00, S D=2.38 \mathrm{sec}$ ).

Control. The mean durations of contacts per object for D4-S1 and D4-S2 were, respectively, $2.44(S D=$ $1.30 \mathrm{sec})$ and $2.81(S D=2.41 \mathrm{sec})$. Individual data are shown in Table 2.

\section{Discussion}

As in the previous experiment, habituation to the initial configuration was present since a decrease of contact duration was observed during that period. Moreover, the spatial modification did not induce a renewal of exploration directed toward the displaced object. The results of the
Habituation

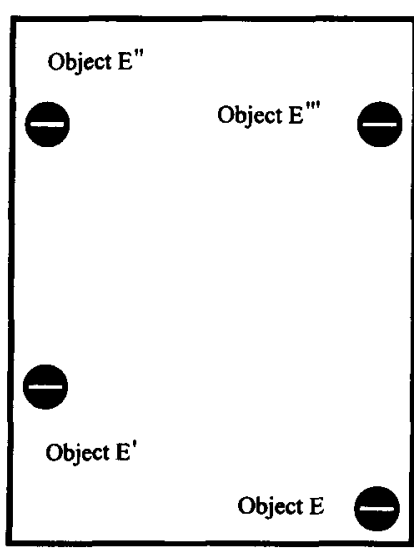

Test

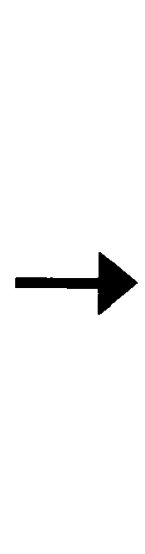

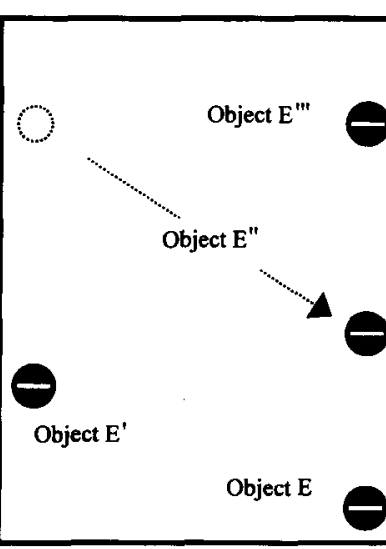

Control

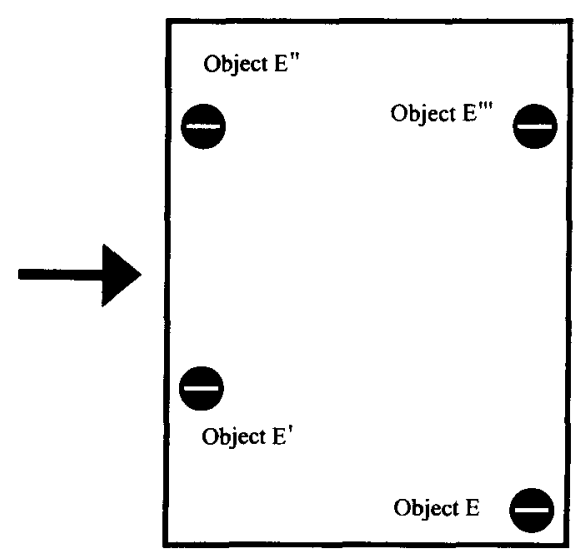

Figure 2. The configuration of the four identical boxes (black circles) in the three phases of Experiment 2. The four objects (E, $E^{\prime}, E^{\prime \prime}$, and $\left.E^{\prime \prime \prime}\right)$ in the boxes are identical (horizontal line). During the test phase, a box was displaced ( $\left.E^{\prime \prime}\right)$ to a new location to change the geometry of the initial configuration. 
Table 2

Experiment 2: Individual Results (Contact Time per Object in Seconds) for the 4 Subjects (Idefix, Ignace, Jade, and Jason)

\begin{tabular}{|c|c|c|c|c|}
\hline \multirow[b]{2}{*}{ Day-Session (D-S) } & \multicolumn{4}{|c|}{ Object } \\
\hline & A & $\mathbf{B}$ & $\mathrm{C}$ & $\mathrm{D}$ \\
\hline \multicolumn{5}{|c|}{ Idefix } \\
\hline 1.1 & 40 & 30 & 27 & 29 \\
\hline $1-2$ & 21 & 20 & 10 & 11 \\
\hline $2-1$ & 4 & 9 & 9 & 4 \\
\hline $2-2$ & 5 & 12 & 8 & 2 \\
\hline $3-1$ & 8 & 11 & 1 & 4 \\
\hline $3-2^{*}$ & 2 & 9 & 7 & 2 \\
\hline $4-1$ & 2 & 1 & 1 & 3 \\
\hline $4-2$ & 1 & 3 & 4 & 2 \\
\hline \multicolumn{5}{|c|}{ Ignace } \\
\hline $1-1$ & 22 & 27 & 21 & 38 \\
\hline $1-2$ & 8 & 14 & 9 & 9 \\
\hline $2-1$ & 6 & 4 & 14 & 4 \\
\hline $2-2$ & 3 & 2 & 4 & 2 \\
\hline $3-1$ & 4 & 1 & 6 & 6 \\
\hline $3.2^{*}$ & 2 & 5 & 3 & 2 \\
\hline $4-1$ & 3 & 0 & 8 & 4 \\
\hline $4-2$ & 1 & 9 & 4 & 6 \\
\hline \multicolumn{5}{|c|}{ Jade } \\
\hline $1-1$ & 24 & 13 & 30 & 28 \\
\hline $1-2$ & 9 & 17 & 21 & 13 \\
\hline $2-1$ & 14 & 9 & 3 & 7 \\
\hline $2-2$ & 3 & 0 & 0 & 5 \\
\hline $3-1$ & 9 & 7 & 4 & 3 \\
\hline $3-2^{*}$ & 5 & 0 & 2 & 0 \\
\hline $4-1$ & 1 & 2 & 3 & 2 \\
\hline $4-2$ & 2 & 0 & 0 & 0 \\
\hline \multicolumn{5}{|c|}{ Jason } \\
\hline $1-1$ & 21 & 24 & 29 & 26 \\
\hline $1-2$ & 15 & 16 & 9 & 11 \\
\hline $2-1$ & 14 & 9 & 7 & 8 \\
\hline $2-2$ & 2 & 3 & 4 & 5 \\
\hline $3-1$ & 7 & 7 & 0 & 9 \\
\hline $3-2^{*}$ & 6 & 0 & 8 & 10 \\
\hline $4-1$ & 1 & 4 & 2 & $\therefore 2$ \\
\hline $4-2$ & 6 & 7 & 0 & 0 \\
\hline
\end{tabular}

*Test Sessions.

control phase indicate that testing conditions did not affect the recollection of the initial configuration by the baboons.

It seems that the spatial change brought about in this experiment was not detected by the animals during the test phase. The local aspect-that is, the object's characteristics - needs to be specified without ambiguity in the spatial representation in order to induce a renewed investigatory response when baboons are confronted with a geometrical modification. To attempt to explain the specific role of the object's characteristics in the reaction-tospatial-change task, we examined, in the next experiment, the impact on the selective reexploration of a local modification without changing the geometrical characteristics of the initial configuration.

\section{EXPERIMENT 3}

The purpose of this experiment was to examine the effect of a substitution of a familiar object with a new one (local modification) without changing the shape of the configuration made by the four objects.

\section{Method}

Animals. The animals were the same 4 baboons (Papio anubis) as in Experiments 1 and 2.

Apparatus. The apparatus was identical to that used in Experiment 1 . The objects chosen were a white wooden board (Object F; $8 \times 8 \mathrm{~cm}$ ), a piece of metal (Object G; $11 \times 9 \mathrm{~cm})$, a blue cup $(\mathrm{Ob}-$ ject $\mathrm{H}$; diameter $10 \mathrm{~cm}$ ), and a green plastic pyramid (Object $\mathrm{I}$; $10 \times 7 \mathrm{~cm})$. All the objects' positions, randomly chosen, were different from those of the previous experiments with respect to their geometric configuration.

Procedure. The basic procedure of Experiment 3 was the same as in the previous experiments. During the test phase (Day 3), Object $\mathrm{H}$ was replaced by a new object, a flower vase (Object $\mathrm{K} ; 12 \times$ $7 \mathrm{~cm}$ ) without changing the spatial configuration of the initial pattern (Figure 3). Experiment 3 was conducted 2 months after the end of Experiment 2 in order to reduce interexperiment interference as much as possible.

\section{Results}

Habituation. As in the previous experiment, the mean duration (in seconds) of contacts per object and per session for all the animals decreased from $26.19(S D=$ $5.84 \mathrm{sec}$ ) in D1-S1 to $3.88[S D=2.61 \mathrm{sec} ; t(15)=8.74$, $p<.001]$ at the end of the habituation session. Furthermore, the mean duration of contacts per object was still longer for the first session (D1-S1:26.19, $S D=5.84 \mathrm{sec}$; D2-S1: $9.19, S D=4.14 \mathrm{sec}$ ) than for the second (D1-S2: $11.06, S D=5.07 \mathrm{sec}$; $22-\mathrm{S} 2: 3.88, S D=2.61 \mathrm{sec})$.

Test (one object changed). The mean duration of contacts for the new object was 5.8 times longer (25.75, $S D=8.63 \mathrm{sec})$ than that for the other objects $(4.42$, $S D=2.39 \mathrm{sec}$ ).

Control. The mean duration of contacts per object for D4-S1 $(15.38, S D=3.30 \mathrm{sec})$ was closer to the duration observed at the beginning of the habituation phase (D1-S1: $26.19, S D=5.84 \mathrm{sec}$ ) than to the contact time observed at the end of habituation phase (D2-S2: $3.88, S D=2.61 \mathrm{sec}$ ). However, the mean contact time during the last session of the control phase (D4-S2: $3.25, S D=3.03 \mathrm{sec}$ ) rapidly reached the level of the end of the habituation phase (D2-S2: $3.88, S D=2.61 \mathrm{sec}$ ). Individual data are presented in Table 3.

\section{Discussion}

As in the previous experiments, a habituation to the initial configuration was present at the end of the habituation phase. The object substitution during the test phase induced a strong exploration specifically directed toward the new object. Moreover, the test sessions seem to have disturbed the recollection of the initial configuration because a renewal of exploration generalized to all objects was observed during the control phase (D4-S1).

This finding underlines the importance of a local modification on the spatial encoding performed by the baboons. If the animals reacted to a local modification, it can be reasonably concluded that the local characteristics of the initial configuration (i.e., the intrinsic character- 
Habituation

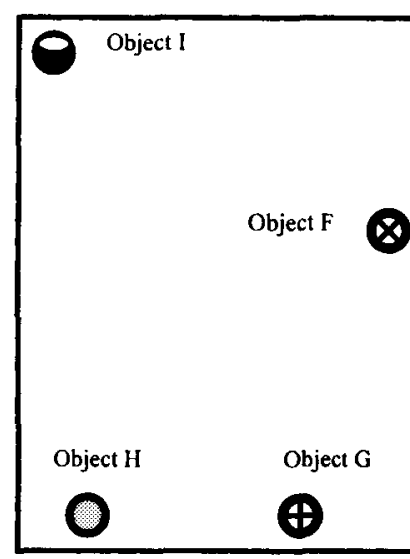

Test

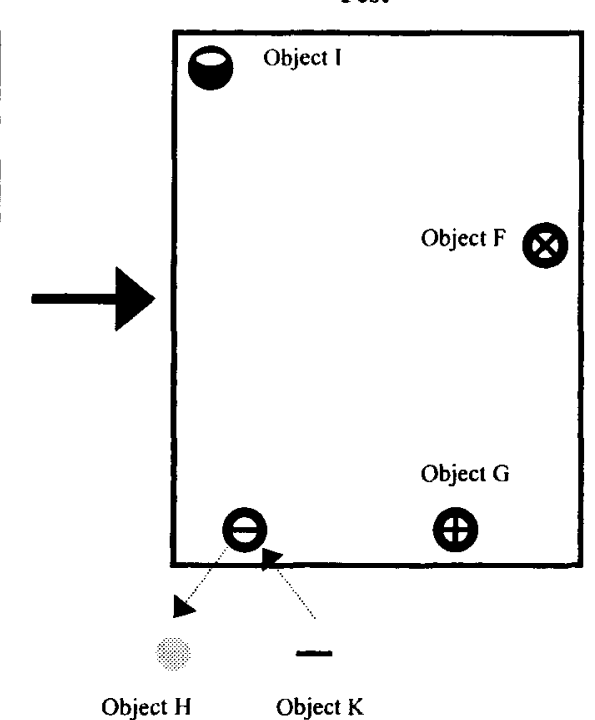

Control

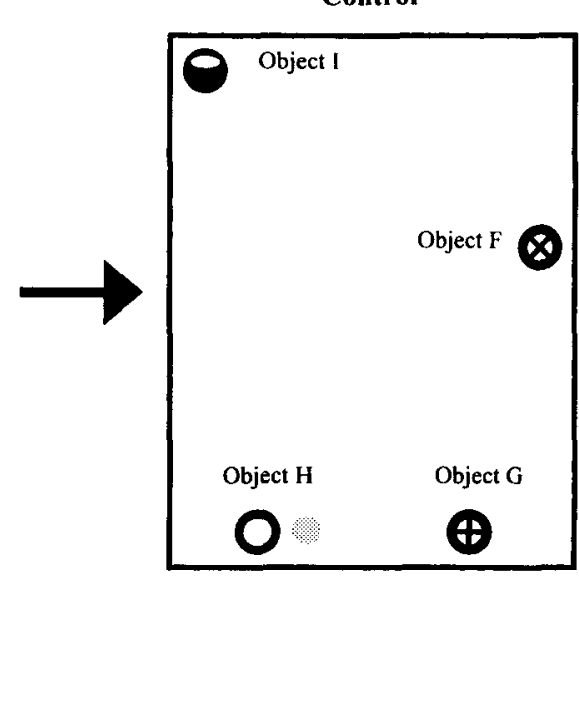

Figure 3. The configuration of the four identical boxes (black circles) in the three phases of Experiment 3 . The different symbols inside the black circles represent the different objects (Objects F, G, H, and I). During the test phase, one object (H) was replaced by a new one.

istics of each object and its position in the configuration) are encoded in the spatial representation of the baboons.

\section{EXPLORATORY STRATEGIES}

For each experiment, for all sessions (except for test sessions) and for each animal, the mean number of visits to the different locations was computed. These data were subjected to a $\chi^{2}$ one-sample test by which we compared a theoretical distribution of equal frequencies of visits to the observed distribution. For each animal, the $\chi^{2}$ value never reached a statistically significant level (at $\alpha=.05$ ). Thus, we can conclude that all places were equally explored by all of the animals.

The total number of visits included in cyclic and backand-forth patterns was divided by the total number of places visited to provide the frequency of visits involved in each category (Figure 4). It appears that $78 \%$ of the visits during Experiments 1-3 were made using either the cyclic or the back-and-forth strategy (Experiment 1 , 74\%; Experiment 2, 91\%; and Experiment 3, 68\%). A simulation of 10,000 randomly chosen visits to the four different locations indicated that $20 \%$ of the visits featured the cyclic strategy and $15 \%$ featured the back-andforth strategy. Results of the exploratory strategies of Experiments $1-3$ were subjected to a $\chi^{2}$ one-sample test by which we compared the theoretical frequency of both strategies to the observed distribution. The results always reach statistical significance $\left(\chi^{2}<.001\right)$. Thus, the pattern of exploration observed during Experiments 1-3 cannot be attributed to chance.

More precisely, in Experiment 1, the use of the backand-forth strategy ( $41 \%$ of visited sites) was higher than the use of the cyclic strategy ( $33 \%$ of visited sites). Conversely, during Experiments 2 and 3, the cyclic strategy was the most frequently used. We observed $52 \%$ of visits using the cyclic strategy, and 39\% using the back-andforth strategy during Experiment 2. For Experiment 3, we noted $51 \%$ of cyclic strategy use and $17 \%$ of backand-forth strategy use.

\section{Discussion}

Our 4 baboons explored several object configurations by using two main locomotor patterns (cyclic and backand-forth). Since the animal exploration was not reinforced and without any initial learning, we assume that the cyclic and the back-and-forth strategies reflect a spontaneous way to become familiar with the spatial characteristics of the environment. The importance and evolution of these two strategies during our experiments will be explored in the general discussion.

\section{GENERAL DISCUSSION}

\section{Detection of Novelty and Spatial Coding}

This study was aimed at providing some information about the spatial cognitive processes employed by baboons to detect and appropriately react to a change in object configuration. In Experiment 1, we examined whether the modification of the shape of the initial configuration induced a renewal of exploration directed toward a displaced object. In Experiment 2, we tested the effect of a spatial modification of an arrangement made by four identical boxes, each one containing an identical object. Finally, in Experiment 3, we examined the effect of a local modification without changing the geometry of the 
Table 3

Experiment 3: Individual Results (Contact Time per Object in Seconds) for the 4 Subjects (Idefix, Ignace, Jade, and Jason)

\begin{tabular}{|c|c|c|c|c|}
\hline \multirow[b]{2}{*}{ Day-Session (D-S) } & \multicolumn{4}{|c|}{ Object } \\
\hline & $\mathrm{A}$ & B & $\mathrm{C}$ & $\mathrm{D}$ \\
\hline \multicolumn{5}{|c|}{ Idefix } \\
\hline $1-1$ & 54 & 18 & 28 & 29 \\
\hline $1-2$ & 21 & 16 & 19 & 18 \\
\hline $2-1$ & 15 & 10 & 5 & 14 \\
\hline $2-2$ & 1 & 2 & 3 & 2 \\
\hline $3-1$ & 5 & 7 & 2 & 4 \\
\hline $3-2^{*}$ & 9 & 11 & 43 & 0 \\
\hline $4-1$ & 15 & 20 & 17 & 11 \\
\hline $4-2$ & 4 & 10 & 6 & 2 \\
\hline \multicolumn{5}{|c|}{ Ignace } \\
\hline $1-1$ & 32 & 23 & 21 & 23 \\
\hline $1-2$ & 18 & 10 & 12 & 14 \\
\hline $2-1$ & 13 & 14 & 13 & 12 \\
\hline $2-2$ & 0 & 2 & 7 & 0 \\
\hline $3-1$ & 3 & 5 & 4 & 2 \\
\hline $3-2^{*}$ & 6 & 4 & 25 & 2 \\
\hline $4-1$ & 23 & 17 & 22 & 18 \\
\hline $4-2$ & 0 & 0 & 8 & 6 \\
\hline \multicolumn{5}{|c|}{ Jade } \\
\hline $1-1$ & 27 & 29 & 21 & 26 \\
\hline $1-2$ & 10 & 9 & 5 & 8 \\
\hline $2-1$ & 2 & 2 & 9 & 11 \\
\hline $2-2$ & 5 & 8 & 9 & 11 \\
\hline $3-1$ & 4 & 2 & 0 & 2 \\
\hline $3-2 *$ & 3 & 1 & 16 & 4 \\
\hline $4-1$ & 12 & 11 & 14 & 9 \\
\hline $4-2$ & 1 & 0 & 0 & 0 \\
\hline \multicolumn{5}{|c|}{ Jason } \\
\hline $1-1$ & 19 & 18 & 20 & 31 \\
\hline $1-2$ & 1 & 4 & 7 & 5 \\
\hline $2-1$ & 13 & 2 & 2 & 10 \\
\hline $2-2$ & 9 & 7 & 2 & 5 \\
\hline $3-1$ & 5 & 3 & 4 & 1 \\
\hline $3-2^{*}$ & 3 & 6 & 19 & 4 \\
\hline $4-1$ & 14 & 15 & 11 & 17 \\
\hline $4-2$ & 8 & 2 & 5 & 0 \\
\hline
\end{tabular}

*Test Sessions.

arrangement. The results of each of these three experiments will be examined in the following section.

The results of Experiment 1 (Test 2) show that baboons are sensitive to a spatial modification of the geometry of an object arrangement. In contrast, animals are less sensitive to a modification that does not affect the shape of the configuration unless the object is novel. It appears that baboons encode the geometrical aspect of the environment only because they do not react to a change that preserves the geometry of the situation. However, the results of Experiment 2 suggest that the geometrical encoding requires that the various elements that define the geometry of the explored space are not identical and that each of them specifies a location. Experiment 3 confirmed that the local aspect of the configuration-that is, the intrinsic characteristics of each object of the configurationis important for the encoding of the whole configuration. This is demonstrated by the finding that a substitution of a familiar object by a new one can be detected without inducing a renewal of exploration generalized to the whole configuration. However, because a renewal of exploration generalized to all objects was observed during the control phase, substitution seems to have disturbed the recollection of the initial configuration. Thus, it appears that a local change to an object arrangement brings about an updating of the initial spatial representation. Nevertheless, the fact that baboons did not react to spatial changes (Experiment 1, Test 1, and Experiment 2) is not an absolute indication of an absence of coding of the characteristics affected by the change. A "nonreaction" to a spatial change can mean either a lack of detection or a detection that does not lead to an overt behavior.

These results indicate that baboons detect and react to spatial modifications of an arrangement of objects. This suggests that an internal model or a cognitive map of the initial situation is built during exploration. This stored internal representation would be systematically compared with a modified presentation of the situation. If the perceived situation differs from the stored model, the animals are likely to notice the modification and to react appropriately so as to update their internal representation. This conclusion is in agreement with previous findings obtained with baboons (Joubert \& Vauclair, 1986), marmosets (Menzel \& Menzel, 1979), and chimpanzees (Menzel, 1978). Nonhuman primates display the ability to become acquainted with the nature and the relative position of objects in their familiar environment. In addition, they demonstrate an excellent ability to rapidly react to new objects among a set of familiar objects.

Our experimental configurations can be defined with respect to two main spatial components: the geometrical aspect of the configuration (global aspect), and the intrinsic features of each site of the configuration (local aspects). Only the geometry of the arrangement can be perceived at distance. The two spatial characteristics (global and local) seem to be encoded in an internal model of the initial situation. Similar to Cheng and Gallistel's (1984) and Cheng's $(1986,1987)$ rats, our baboons primarily used, while orienting in space, "a purely geometric module," namely a representation that predominantly encodes the geometrical shape of the environment. According to these authors, this single modular treatment of spatial information does not specify the location of the local characteristics of the environment. The environmental features are encoded in other modules that are specifically bound to the geometrical module via "address labels." Each local characteristic of the environment is encoded with an address label specifying a position in the geometrical module and, conversely, the locations in the geometrical module might also carry labels specifying what local characteristics are found at those addresses. Presence of a geometric module has also been described in birds (Vallortigara, Zanforlin, \& Pasti, 1990) and in human infants (Hermer \& Spelke, 1994, 1996). Thus, it seems that the capacity to encode and process the geometric characteristics of the environment are present not only throughout the animal phylogeny but also early in the cognitive development of animals. Furthermore, our experiments provide some evidence that baboons are 


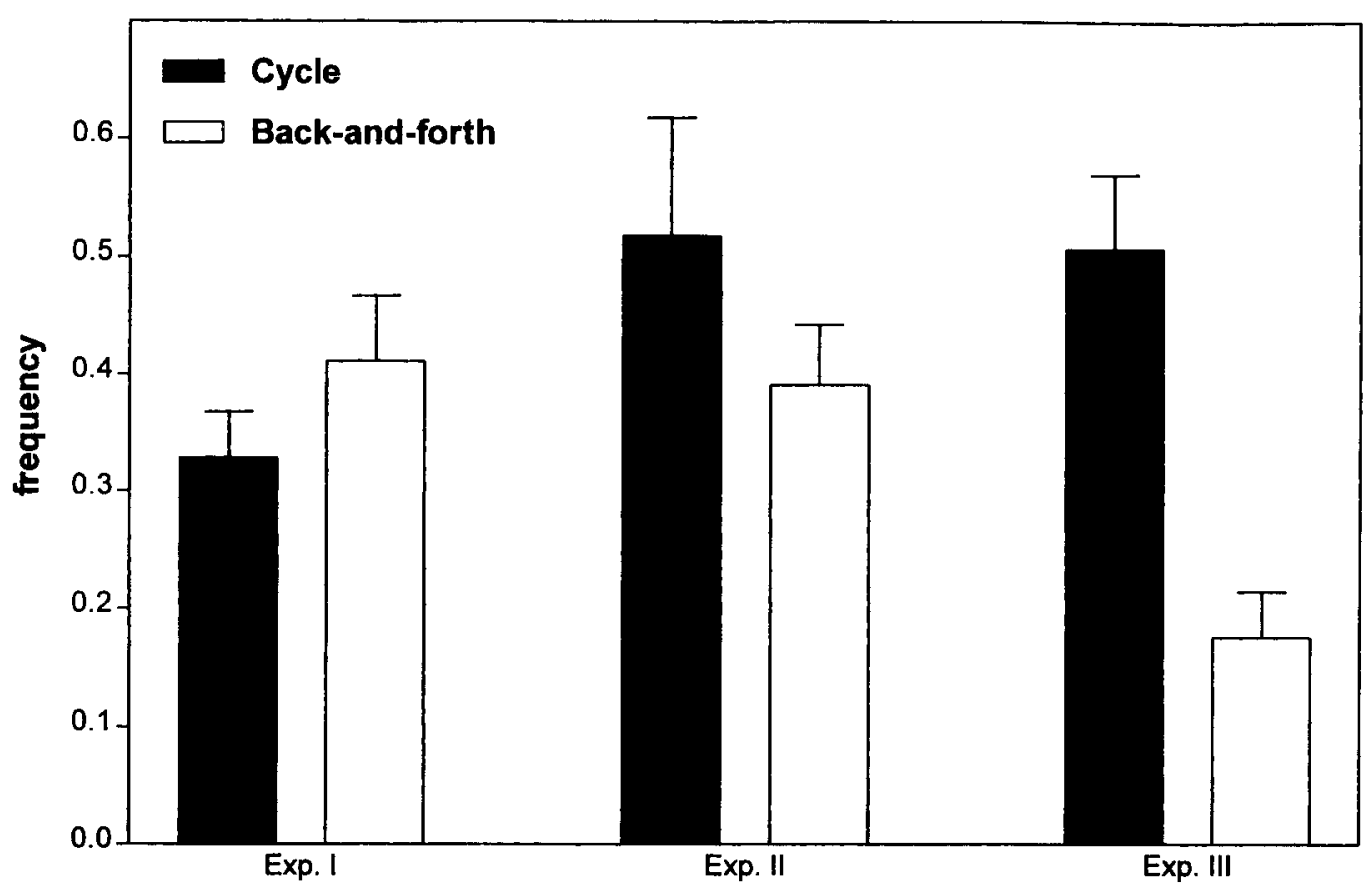

Figure 4. Frequency of the visits included in the cyclic and the back-and-forth patterns for all the experiments, and for all the animals.

also able to react to featural modifications of an object configuration, if all the objects are different. When the objects were identical, no renewal of exploration was observed when animals were confronted with a geometrical modification (Experiment 2). This suggests that featural information is taken into account in the elaboration of the geometrical module hypothesized by Cheng (1986). An alternative interpretation is that a general process of habituation may have taken place from Experiment 1 to Experiment 2, precluding reaction to novelty. However, given that (1) the habituation level at the beginning of the exploratory phase was similar in Experiment 2 (and Experiment 3) compared with Experiment 1, (2) the rates of habituation were comparable across the exploratory sessions within each experiment, and (3) each experiment was separated by a 2 -month period, we can reasonably assume that interexperiment habituation cannot explain the subjects' lack of reaction in Experiment 2.

\section{Exploratory Strategies}

Two kinds of locomotor regularities were observed in our baboons during the exploration of the configuration of four objects: a cyclic pattern and a back-and-forth pattern. These patterns represented $78 \%$ of the visits to the different sites during the three experiments $(74 \%$ in Experiment 1, 91\% in Experiment 2, and 68\% in Experiment 3 ). The remaining $22 \%$ involved nonordinate visits to the different places (e.g., many visits to the same place) and were mainly observed toward the end of the habituation phase and in the control phase. Thus, the cyclic and the back-and-forth strategies were the pre- dominant means for processing unfamiliar spatial information. Spatial representations, according to Neisser's (1976) theory, "direct" perceptual exploration, which, in turn, modifies initial representations. The strategies observed during that exploration should thus reflect some preexisting organizing schemata that are dependent on the characteristics of the situation to be encoded (see Thinus-Blanc \& Gaunet, 1998).

From the data obtained on humans (Gaunet $\&$ ThinusBlanc, 1996), we hypothesize that the cyclic strategy observed in our baboons might be taken as corresponding to the learning of the overall configuration of locations, whereas the back-and-forth pattern would correspond to a precise estimation of distance and angle relationships between the various objects of the configuration. That hypothesis would explain why we observed a decrease in the use of the back-and-forth strategy and an increase of the cyclic strategy from Experiment 1 to Experiment 3. Animals may have adapted their exploratory behavior during the tests so as to progressively optimize their behavior by taking into account, during exploration, the geometry of the configuration. Thus, baboons, like capuchin monkeys (De Lillo,Visalberghi, \& Aversano, 1996, 1997) seem to regulate and structure their spatial behavior during exploration.

Another hypothesis might explain the changes in the use of the exploration strategies in our experiments. According to Menzel (1973) and Cramer and Gallistel (1997), primates are able to optimize their exploratory trajectories in order to minimize the distance traveled when they are confronted with baited sites. In the present experi- 
ments, even if the animals were not reinforced during exploration, it seems that they learned to use the most "economical" strategy to explore the whole configuration. Our baboons progressively used the cyclic strategy presumably because it reduces the total distance traveled to visit all the sites of the experimental situation. For example, if we consider a rectangular enclosure of $20 \mathrm{~m}^{2}$ $(5 \times 4 \mathrm{~m})$ with four objects $(\mathrm{A}, \mathrm{B}, \mathrm{C}$, and $\mathrm{D})$, one in each corner, then the use of a cyclic strategy (ABCDA) will lead to a total distance of $18 \mathrm{~m}$ to visit all the sites, whereas the use of a back-and-forth strategy (ABACADA) will lead to a total distance traveled of about $24.4 \mathrm{~m}$. Thus, spatial exploration may be under the control of mechanisms aimed at optimizing not only the energy cost, but also the cognitive cost of exploration.

In conclusion, the present findings suggest that the baboons encode the two main spatial characteristics of the configuration of objects with which they are confronted: the global geometry and the local characteristics (nature and position of each object). Furthermore, the geometric and the local aspects of the configuration appear to be hierarchically organized in the baboon's spatial representation. In effect, the monkeys appear to be more sensitive to geometric modifications than to local modifications (without change in the shape of the configuration). According to O'Keefe and Nadel's (1978) theory, exploration owing to locomotion allows organisms to build spatial representations of their environment. It can be added that exploration is not random but rather is under the control of mechanisms that direct the acquisition of the characteristics defining space. Thus, the cognitive processes involved in the elaboration and use of spatial representations appear to be highly structured. Baboons must take into account the acquisition of spatial information (exploration by way of exploratory strategies) for the elaboration of an internal representation (cognitive mapping). All those processes might be devoted to one main objective: the optimization of exploratory trajectories (e.g., the traveling salesman problem). Thus, study of the factors that account for a particular exploratory strategy will likely contribute to a better understanding of the mechanisms involved in spatial cognition.

\section{REFERENCES}

BERLYNE, D. E. (1960). Conflict, arousal, and curiosity. New York: McGraw-Hill.

Brodbeck, D. R. (1994). Memory for spatial and local cues: A comparison of a storing and a nonstoring species. Animal Learning \& Behavior, 22, 119-133.

Cheal, M. L. (1978). Stimulus-elicited investigation in the Mongolian gerbil (Meriones unguiculatus). Journal of Biological Psychology, 20, 26-30.

ChEnG, K. (1986). A purely geometric module in the rat's spatial representation. Cognition, 23, 149-178.

CHENG, K. (1987). Rats use the geometry of surfaces for navigation. In P. Ellen \& C. Thinus-Blanc (Ed.), Cognitive processes and spatial orientation in animals and man (pp. 153-159). Dordrecht: Martinus Nijhoff.
Cheng, K., \& Gallistel, C. R. (1984). Testing the geometric power of an animal's spatial representation. In H. L. Roitblat, T. G. Bever, \& H. S. Terrace (Eds.), Animal cognition (pp. 409-423). Hillsdale, NJ: Erlbaum.

Corman, C., \& Shafer, J. (1968). Open-field activity and exploration. Psychonomic Science, 13, 55-56.

CRAmer, A., \& Gallistel, C. (1997). Vervet monkeys as travelling salesmen. Nature, 387, 464.

De Lillo, C., Visalberghi, E., \& Aversano, M. (1996). The economy of capuchin monkey search behaviour in small- and large-scale environments. Folia Primatologica, 67, 2, 64-65.

De Lillo, C., Visalberghi, E., \& Aversano, M. (1997). The organization of exhaustive searches in a patchy space by capuchin monkeys (Cebus apella). Journal of Comparative Psychology, 111, 82-90.

Gaunet, F., \& Thinus-Blanc, C. (1996). Early blind animal's spatial abilities in the locomotor space: A study of exploratory strategies and reaction to change performance. Perception, 25, 967-981.

Hermer, L., \& Spelke, E. (1994). A geometric process for spatial reorientation in young children. Nature, 370, 57-59.

Hermer, L., \& SPelke, E. (1996). Modularity and development: The case of spatial reorientation. Cognition, 61, 195-232.

Joubert, A., \& Vauclair, J. (1986). Reaction to novel objects in a troop of Guinea baboons: Approach and manipulation. Behaviour, 96, 92-104.

MACDONALD, S. (1994). Gorillas' (Gorilla gorilla gorilla) spatial memory in a foraging task. Journal of Comparative Psychology, 108, 107-113.

MacDonald, S., Pang, J., \& Gibeault, S. (1994). Marmoset (Callithrix jacchus jacchus) spatial memory in a foraging task: Win stay versus win-shift strategies. Journal of Comparative Psychology, 108, 328-334.

MacDonald, S., \& Wilkie, D. (1990). Yellow-nosed monkeys' (Cercopithecus ascanius whitesidei) spatial memory in a simulated foraging environment. Journal of Comparative Psychology, 104, 382-387.

MENZEL, E. W. (1973). Chimpanzee spatial memory organization. Science, 182, 943-945.

Menzel, E. W. (1978). Cognitive mapping in chimpanzees. In S. H. Hulse, H. Fowler, \& W. K. Honig (Eds.), Cognitive processes in animal behavior (pp. 375-422). Hillsdale, NJ: Erlbaum.

Menzel, E. W., \& Menzel, C. R. (1979). Cognitive developmental and social aspects of responsiveness to novel objects in a family group of marmosets (Saguinus fuscicollis). Behavior, 70, 251-278.

NeISSER, U. (1976). Cognition and reality. San Francisco: W. H. Freeman.

O'KeEFE, E. W., \& NADEL, L. (1978). The hippocampus as a cognitive map. London: Oxford University Press.

Poucet, B., Chapuis, N., Durup, M., \& Thinus-Blanc, C. (1986). A study of exploratory behavior as an index of spatial knowledge in hamsters. Animal Learning \& Behavior, 14, 93-100.

Thinus-Blanc, C., Bouzouba, L., Chaix, K., Chapuis, N., Durup, M., \& PoucET, B. (1987). A study of spatial parameters encoded during exploration in hamsters. Journal of Experimental Psychology: Animal Behavior Processes, 13, 418-427.

Thinus-Blanc, C., \& GaunEt, F. (1998). Spatial processing in animal and man. The two-fold function of spatial representations. In R. G. Golledge (Ed.), Wayfinding: Cognitive mapping and spatial behavior (pp. 294-307). Baltimore: John Hopkins University Press.

TinKLEPAUGH, O. (1932). Multiple delayed reaction with chimpanzees and monkeys. Journal of Comparative Psychology, 13, 207-243.

Tolman, E. C. (1948). Cognitive maps in rats and men. Psychological Bulletin, 55, 189-208.

Vallortigara, G., Zanforlin, M., \& Pasti, G. (1990). Geometric modules in animals' spatial representations: A test with chicks (Gallus gallus domesticus). Journal of Comparative Psychology, 104, 248-254.

(Manuscript received August 31, 1998; revision accepted for publication January 7, 1999.) 